\title{
LAS ACOTACIONES DE ROSSO DI SAN SECONDO A SU MARIONETTE, CHE PASSIONE!
}

\author{
ana Martínez-Penuela Vírseda \\ Universidad Complutense de Madrid
}

Al enfrentarnos a un texto teatral no podemos olvidar que el texto unitario del espectáculo, como señala Lotman', está formado por tres «subtextos", cada uno con una cierta independencia: el texto verbal de la obra, el texto escénico creado por el director y por los actores y el pictórico musical. Estos subtextos llegan a constituir una unidad, pero el proceso de codificación actúa de distinta manera para cada uno de ellos.

En el texto escénico cada eslabón de la cadena de la creación del texto (desde el actor al director, al técnico de iluminación, etc...) está sujeto a un doble comportamiento. Por un lado no hay libertad. El autor está determinado por la tradición, por los gustos del público, por las ideas de la época; el director, por su parte, está mediatizado por las intenciones del autor, por la censura, el empresario, etc... Sin embargo, cada eslabón de la cadena no se limita sólo a la ejecución, sino también a la labor creativa común y ahí es donde está la libertad. El espectáculo no es la realización de un programa único, ya dado, sino que actúa a través de un conflicto cuyos resultados no se pueden prever de un modo estricto y ésto es precisamente lo que le da al texto escénico un enorme potencial de significado.

En una obra teatral distinguiremos texto literario y texto espectacular como dos aspectos de un único proceso de comunicación. En general texto literario será el texto dialogado, abarcando también a veces prólogo, acotaciones, etc... y texto espectacular el conjunto de indicaciones y sugerencias que proceden de las acotaciones y a veces del mismo diálogo.

El texto literario va dirigido a la lectura y el texto espectacular a la

' J. Lotman, «Semiótica della scena», en Strumenti critici, 44, febbraio 1981, pp. 19-20. 
representación, si bien ambos están en el texto escrito. Un texto literario es teatral en la medida en la que nace ya destinado a ser espectáculo ${ }^{2}$.

Como claramente explica $\mathrm{M}^{\mathrm{a}}$ del Carmen Bobes ${ }^{3}$ «una semiología del teatro no puede plantear dialécticamente el estudio del texto y el de la representación (...), ya que son formas de recepción distintas en un proceso de comunicación único en el que todo se organiza en una unidad de lectura, en una unidad de representación".

Aunque el hecho teatral resulte de la articulación de los dos textos y aunque en el texto escrito haya índices del texto escénico, creo que es posible, en un principio, analizar por separado los dos textos, ya que, como dice Díez Borque, el escenario es el contexto del texto escrito en el que se pretende que "unos significantes insuficientes, o que inmediatamente significan otra cosa, signifiquen lo que se quiere que signifiquen según las necesidades - del texto escrito ${ }^{4}$, con lo cual ambos textos intercambian sus funciones: el signo lingüístico como signo escénico.

Para nuestro análisis partiremos del texto escrito, el texto dramático en el que podemos distinguir dos partes: el texto dialogado y el texto de las acotaciones, rechazando la denominación de Ingarder de texto principal (diálogo) y texto secundario (acotaciones), por lo que tiene de valorativo, ya que en este caso concreto el texto de las acotaciones es tan "principal» como el del diálogo, sin olvidar que en el texto dialogado pueden aparecer referencias a otro tipo de signos no lingüísticos que ayudarán a una mejor puesta en escena, si bien será el texto de las acotaciones más el texto dialogado el que nos dé una visión total y globalizadora de la obra.

En el texto escrito las acotaciones se dirigen al lector y sugieren una construcción imaginaria de la obra, de los personajes y su actuación, de tiempos, espacios, etc...

Las acotaciones del texto escrito conducen necesariamente a su ejecución espectacular. De Marinis ${ }^{5}$ explica la irreversibilidad del proceso texto dramático $\rightarrow$ espectáculo y por consiguiente acotación escrita $\rightarrow$ acotación transcodificada como consecuencia del lenguaje non-notazionale de las acotaciones. Habla de la imposibilidad de retroceder de la transcodificación espectacular (la ejecución) de una cierta acotación a la acotación misma.

Una acotación transcodificada en el espectáculo puede ser congruente con diversas acotaciones escritas y, al revés, una misma acotación dramática puede tener más transcodificaciones espectaculares, todas ellas congruentes.

2 Sobre este tema cfr. F. RuFFINI, "Testo letterario, testo spettacolare», en Semiótica del testo, L'esempio teatro, Roma, Bulzoni, 1978.

3 M. C. Bobes, Semiología de la obra dramática, Madrid, Taurus, 1987, p. 29.

- J. M. Diez Borque, "Aproximación a la "escena" del teatro del Siglo de Oro" en AA.VV., Semiologia del teatro, Barcelona, Planeta, 1975, p. 54.

${ }^{3}$ M. De MARInis, Semiotica del teatro. L'analisi testuale dello spettacolo, Milano, Bompiani, 1982, p. 39. 
Esto significa que por un lado no es posible reconstruir la parte de las acotaciones de un texto dramático partiendo de una transcodificación espectacular y por otro tampoco es posible verificar si una cierta acotación ha sido transcodificada correctamete; dependerá de la interpretación que se les dé.

A pesar de este último riesgo vamos a tratar de hacer un análisis del texto del espectáculo, tendiendo a una definición sistemática de éste, para lo cual aludiré a los sistemas de signos que Tadeusz Kowzan ${ }^{6}$ encuentra en el teatro (dejando a un lado la palabra, el diálogo, que puede ser objeto de otro estudio monográfico), por considerarla una propuesta eficaz para el estudio de los códigos teatrales.

\begin{tabular}{|c|c|c|c|c|c|}
\hline $\begin{array}{l}1 \text { la palabra } \\
2 \text { tono }\end{array}$ & $\begin{array}{l}\text { Texto pro- } \\
\text { nunciado }\end{array}$ & \multirow{3}{*}{ Actor } & $\begin{array}{l}\text { Signos } \\
\text { auditivos }\end{array}$ & Tiempo & $\begin{array}{l}\text { Signos au- } \\
\text { ditivos } \\
\text { (actor) }\end{array}$ \\
\hline $\begin{array}{l}3 \text { mimica } \\
4 \text { gesto } \\
5 \text { movimiento }\end{array}$ & $\begin{array}{l}\text { Expresión } \\
\text { corporal }\end{array}$ & & \multirow{3}{*}{$\begin{array}{l}\text { Signos } \\
\text { visuales }\end{array}$} & $\begin{array}{l}\text { Espacio } \\
\text { y tiempo }\end{array}$ & \multirow{2}{*}{$\begin{array}{l}\text { Signos vi- } \\
\text { suales } \\
\text { (actor) }\end{array}$} \\
\hline $\begin{array}{l}6 \text { maquillaje } \\
7 \text { peinado } \\
8 \text { traje } \\
\end{array}$ & $\begin{array}{l}\text { Apariencias } \\
\text { exteriores } \\
\text { del actor }\end{array}$ & & & Espacio & \\
\hline $\begin{array}{l}9 \text { accesorios } \\
10 \text { decorado } \\
11 \text { iluminación } \\
\end{array}$ & $\begin{array}{l}\text { Aspecto } \\
\text { del espacio } \\
\text { escénico }\end{array}$ & \multirow{2}{*}{$\begin{array}{l}\text { Fuera } \\
\text { del actor }\end{array}$} & & $\begin{array}{l}\text { Espacio } \\
\text { y tiempo }\end{array}$ & $\begin{array}{l}\text { Signos vi- } \\
\text { suales } \\
\text { (fuera del } \\
\text { actor) }\end{array}$ \\
\hline $\begin{array}{l}12 \text { música } \\
13 \text { sonido }\end{array}$ & $\begin{array}{l}\text { Efectos } \\
\text { sonoros no } \\
\text { articulados }\end{array}$ & & $\begin{array}{l}\text { Signos } \\
\text { auditivos }\end{array}$ & Tiempo & $\begin{array}{l}\text { Signos au- } \\
\text { ditivos } \\
\text { (fuera del } \\
\text { actor) }\end{array}$ \\
\hline
\end{tabular}

Algunos de estos signos han adquirido independencia al establecerse dentro de las disciplinas semióticas como la «kinésica» (gesto), proxémica (movimiento) y paralingüística (tono).

6 T. KowZAN, «El signo en el teatro. Introducción a la semiologia del arte del espectáculo", en AA.VV., El teatro y sus crisis actual. Caracas, Monte Avila, 1969, p. 52. 
Creemos que en la obra del autor siciliano Pier Maria Rosso di San Secondo, Marionette, che passione?, las acotaciones tienen un gran valor, por lo que un análisis de éstas nos llevará hacia una más precisa aproximación al texto que nos quiso transmitir su autor.

En Marionette, che passione! podemos distinguir, por un lado, un discurso más directo que el autor dirige al destinatario lector (o espectador si éste se leyese en escena) materializado en el «Preludio» que forma parte integrante de la obra y que a su vez procede de un cuento anterior del mismo autor titulado Acquerugiola. Por otro lado, la indirecta comunicación que es la mímesis que se desarrolla en el escenario. El «Preludio», como los prólogos de las obras antiguas, es el lugar privilegiado en el que el yo emisor puede hablar de forma directa con el yo destinatario. Además Rosso di San Secondo precede este «Preludio» de una "Avvertenza per gli attori» en la que se dice

"Tengano presente gli attori che questa è una commedia di pause disperate... Le parole che vi si dicono celano sempre una esasperazione che non può essere resa se non in sapienti silenzi. L'arbitrario, inoltre, che può parere vi sia nella commedia, risultando dal tormento in cui si macerano i personaggi, non deve dar luogo al comico, bensi a un sentimento di tragico umorismo. Pur soffrendo, infatti, pene profondamente umane, $i$ tre protagonisti del dramma, specialmente, sono come marionette, e il loro filo è la passione. Sono tuttavia uomini: uomini ridotti marionette.

$\mathrm{E}$, dunque, profondamente pietosi!» ${ }^{8}$.

En el «Preludio» que sigue establece el autor el tono preciso y esencial en el que deben comunicarse los actores (los sabios silencios) y resume también la problemática fundamental de la obra, en pocas pero significativas palabras (las marionetas movidas por el hilo de la pasión), tratando de conectar humanamente con los actores, y haciendo entender al destinatario aquello que esencialmente quiere transmitir en su obra.

El resto del «Preludio» recoge las reflexiones de Rosso sobre el modo de ver la vida que quiere condensar en su breve obra. En las largas tardes de domingo, en el breve tiempo de ocio del que el hombre dispone, Rosso reflexiona sobre si mismo, sobre su existencia, que es generalmente soledad, aburrimiento, angustia, incomunicación. Es una visión oscura de la vida en

' Seguimos la edición de L. Ferrante, Marionette, che passione!, en P. M. Rosso di San Secondo, Teatro, Roma, Bulzoni, 1976, vol. I, pp. 129-165.

Ibidem., p. 29. 
la que la libertad, las posibilidades, están abolutamente limitadas. Veamos un ejemplo:

«Oh, letto di domenica sera con il numero del chilometro segnato a piè! Bisognerà domani alzarsi presto di nuovo per ricominciare, se nella notte pian piano il destino non cambierà il cartello o sotto il numero scriverà "ultimo" $"$ ".

Frente a los que han conseguido un sistema de vida estable, en familia, están los otros, "gli sperduti del mondo, i senza casa, i vagabondi..., i randagi della vita» que luchan contra «le inesorabili barriere delle umane possibilità».

El autor reflexiona sobre todo esto y pasa luego, al final del «Preludio», a colocarse de espectador ( $y$ también protagonista) frente al escenario:

"Aggiustandomi la cravatta, che minaccia irragionevoli smanie, mi abbottono nel mio pastrano, e sento, non senza legittima soddisfazione, che l'amido del colletto frena le irrequiete corde della mia nuca, le quali vorrebbero, tirando in tutti $i$ versi, comunicare al mio capo non so che ambigui moti burattineschi, con di più smorfie di viso e boccacce che indignerebbero il rispettabile pubblicon" ${ }^{10}$.

Dentro de las acotaciones, como ya es sabido, podemos distinguir cuatro funciones lingüísticas: referencial, meta-lingüística, perlocutoria y poética. De éstas resaltaremos en primer lugar, en la obra de Rosso, la belleza estética dominante en el «Preludio», como portadora de la poética específica del autor, asumiendo así un espesor semántico de decisiva importancia. En esta extensa acotación para el lector, el director y los actores, queda a un lado su función referencial: más que dar órdenes para la preparación de la escena, revela y prepara la dimensión interna de los personajes, indicando el preciso ambiente espiritual en el que deberán situarse.

Por otra parte podemos también destacar, en los tres actos que constituyen el texto, la función referencial de las acotaciones, con la particula-

9 L. Ferrante, Marionette, che passione!..., op. cit., p. 130.

${ }^{10}$ Ibidem., p. 132. 
ridad de que se trata de indicaciones escénicas que presuponen una relación internà entre acotación y acción interna no habitual hasta entonces, reflejo del expresionismo alemán. No olvidemos que Rosso entre 1926 y 1932 hizo largos y frecuentes viajes a Alemania por motivos de trabajo y también, quizás, para evadirse del ambiente que se respiraba en su país. En Alemania se preocupó de estudiar y conocer esos fermentos literarios que tanto han contribuido a los aspectos expresionistas de su teatro"

En el teatro expresionista, y en el de Rosso, se da una intensa relación entre texto y representación ${ }^{12}$; el diálogo se apoya en las acotaciones y éstas precisan de la funcionalidad de todos los signos del texto. Rosso propone en la escena lo que es el presupuesto fundamental del expresionismo: la visión interna de la realidad como única verdad del alma y de las cosas.

En las acotaciones de los tres actos de Marionette, che passione! se ofrecen al director y al lector indicaciones precisas sobre los objetos, las luces $\mathrm{y}$ todos los componentes que se presentan en el escenario, ya que a diferencia del «Preludio» en estas acotaciones son pocos los valores referenciales que no pueden traducirse en objetos.

Pasemos ya a señalar los sistemas de signos determinados por Kowzan dejando, como ya hemos dicho, a un lado el análisis de la palabra, del diálogo, si bien en el texto literario se pueden encontrar referencias a otros sistemas de signos no verbales.

\section{TEXTO PRONUNCIADO}

En Marionette, che passione! Rosso por una parte adopta la actitud de un espectador más, sin dar órdenes directas para preparar y disponer el escenario, diciéndonos lo que hay, no lo que debe haber, aunque eso presuponga la realización de todas las referencias que existen en el texto. Esto lo podemos deducir por la permanente utilización de verbos en presente indicando que las acciones transcurren en el tiempo de la representación, compartida por autor y espectador. Sin embargo, por otra parte, Rosso no abandona la autoría y dirección de la obra dando ya en la primera acotación la siguiente orden a los actores: "L'intero atto sarà recitato a bassa voce...", lo que demuestra la importancia que el autor da al tono para crear ese

1 Cfr. P. Chiarini, "Rosso di San Secondo e il teatro tedesco del Novecento», en Studi germanici, III, 1, 1965, pp. 90-117 y n. 3, 1965, pp. 321-355.

12 A. Martinez-Peñuela, "Rasgos expresionistas en Rosso di San Secondo y Valle-INCLÁN", en Actas del V Congreso de Italianistas Españoles, Universidad de Oviedo, 1990. 
ambiente de penumbra en el que viven sus personajes, teniendo en cuenta que ésta será la única orden que aparece a lo largo de toda la obra. Rosso crea un mundo rico de significados e intenciones en las acotaciones referidas al tono.

En el acto primero son muchas las referencias que se hacen a él: «a bassa voce / bisbigliando / a parlare sommessamente / piano sempre», etc..., de las que sólo una "a voce alta" indica un cambio respecto al silencio que predomina en todo el acto. El tono, en este acto, contribuye a crear ese ambiente de penumbra, silencio y somnolencia que necesita el autor para la presentación de los personajes.

En los otros dos actos, las escasas indicaciones que encontramos referentes al tono están en relación con el contenido del diálogo, con la actitud del personaje, asi por ejemplo, «Il Signore in grigio» unas veces utilizará un tono irónico, otras «angoscioso, straziante», pero siempre «senza perdere il suo sangue freddo", lo que no le permitirá tantas convulsiones y estridencias como a los otros dos personajes, aunque le vemos en el segundo acto jugando con su propio dolor, un terrible juego de ficción y verdad, cambiando el tono, la voz y los modos según la parte que interprete y obteniendo así sorprendentes transformaciones durante todo el acto.

\section{ASPECTO DEL ESPACIO ESCÉNICO}

Pasaremos a ocuparnos de los signos que según este esquema de Kowzan están fuera del área del actor y que servirán para indicar el aspecto del espacio escénico en el que se va a desarrollar la acción: decorado, accesorios e iluminación.

El decorado comprende no sólo formas sino también luz y color, y está formado por una serie de signos cuyos significados son muy diversos.

Kowzan afirma que el valor semiótico de un decorado no depende directamente de la cantidad de signos de primer grado, ya que un signo aislado puede tener un contenido semántico más rico y denso que todo un conjunto de signos $^{13}$.

En esta obra la mayoria de los signos escénicos están perfectamente claros como para que no sea posible imaginar algo distinto de lo que en realidad el autor quiere que signifiquen. El autor se ha preocupado de describir el espacio escénico necesario para el desarrollo de la obra en cada uno de los tres actos.

\footnotetext{
${ }^{13}$ T. KowZAN, op. cit., p. 46.
} 
Al inicio de cada acto hay una acotación que sitúa la acción en un lugar determinado de la ciudad de Milán. Aunque como es lógico llegaremos a saber que se trata de Milán a través del diálogo y no de las acotaciones. El que la acción se desarrolle en Milán no es casual, Rosso amaba esa ciudad y había descubierto que ésta era un punto de observación que le permitía tomar de la realidad todo lo que de nuevo fermentaba en el mundo, era una ciudad moderna y su oficina de correos le sugirió el ambiente para su primera comedia.

Veamos la acotación que introduce el primer acto, con un claro valor descriptivo:

«La sala del Telegrafo centrale di Milano, in un pomeriggio domenicale. Penombra. Da sinistra verso il fondo corre la vetrata con gli sportelli, di cui due soli sono aperti. In fondo s'indovina, più che non si veda, la porta d'entrata. A destra, parete con manifesti, affissi, prescrizioni.

Sul davanti, verso sinistra, una larga tavola per la scrittura dei telegrammi. Piú in fondo, verso destra, una tavola piccola, per la guardia. Silenzio e sonnolenza. Fuori il tempo è uggioso: pioviggina. L'intero atto sarà recitato a bassa voce con lunghe pause» ${ }^{14}$.

Rosso nos coloca en la Sala de Telégrafos, describe la distribución del decorado, pero sobre todo presenta un ambiente propicio para el desarrollo del comportamiento psicológico de esos personajes concretos: «penombra, silenzio e sonnolenzia" que contrasta con el desarrollarse de un encuentro y un acontecimiento humano extraordinario. La acotación tiene la función de revelar y preparar la dimensión interna de los personajes, colocándoles en un ambiente espiritual adecuado.

El lugar está perfectamente elegido por Rosso como índice de espacio público anónimo, es en teoría el centro de comunicación con los de fuera y en este caso, sobre todo, con los de dentro del escenario. La Sala de Telégrafos va a servir de lugar de encuentro casual de marionetas atormentadas que les va a permitir comunicarse dentro de su soledad.

En el espacio teatral de este acto sólo hay una puerta que no se ve, por donde entran y salen los actores, y no hay ninguna ventana por donde el paso de la luz pueda reflejar esa penumbra de una tarde lluviosa de domingo. El espectador tendrá que deducirlo por la vestimenta y el diálogo

14 Rosso di SAN SeCONDO, op. cit., p. 133. 
de los personajes que van entrando y, sobre todo, por el tono de voz que utilizan.

Habrá también en escena una mesa larga para escribir los telegramas, en torno a la cual se sentarán los tres personajes y que en este caso servirá para indicar al espectador la proximidad física o distanciamiento entre ellos. Esta mesa, a su vez, la podemos considerar como un escenario dentro de la escena (el teatro en el teatro) desde cuya punta "Il Signore in grigio» (personaje que encarna el yo-épico de Rosso) observa, como espectador, a los otros dos personajes al mismo tiempo que dirige la acción.

En el segundo acto se pasa en cambio a describir no un lugar público, sino uno privado:

«Salottino di un appartamento che s'affitta a stanze mobiliate. Dà, a prima giunda, la sensazione del reccattato, del logoro per troppo uso: poltrocione, sofà, tende, tappeti sbiaditi. Mobili senza stile. Una porta in fondo, una a destra.

A sinistra un pianoforte: accanto, uno sgabello con un mucchio di carte di musica. In fondo, a destra, un étagère con uno specchio. Avanti, a destra, una scrivania. Un'ora dopo il primo atto. E sera. Lampadine centrali accese» ${ }^{15}$.

Se trata de la casa donde vive "La Cantante», en donde todos los accesorios puestos por Rosso en escena cumplen su función: el sofá sirve para presentar a la "Signora dalla volpe azzurra» tumbada, desmadejada, destrozada por tanto sufrimiento. El piano con las partituras nos situarán en un escenario en donde aparecerán "La Cantante» y algunas bailarinas que darán un aire festivo a todo el acto. La escribanía tiene el valor icónico de establecer un contraste entre la soledad, sufrimiento, incomunicación de la "Signora dalla volpe azzurra" y la alegría y comunicabilidad de una bailarina a través de los folios y el sobre que están en el escritorio. Y en torno al tocador con el espejo se desarrolla la última parte de este acto que dará lugar a la continuación de la obra.

El espacio estará limitado por dos puertas, una que comunica con el interior, por donde se presentarán los personajes que viven en la casa, y otra que comunica con el exterior por donde entrarán en escena los de fuera.

En el tercer acto la acción se desplaza a una

is Rosso di San Secondo, op. cit., p. 144. 
"Saletta appartata, in una trattoria di lusso. Soffitto stuccato a fiorami con filetti d'oro: pareti di seta gialla, nude. Lampadario centrale. Passaggio in fondo, a destra, nascosto da una tenda di seta, parimenti gialla. Finta porta a sinistra simulata da tenda uguale. Due tavole quadrate, bianchissime, scintillanti di cristalli: una a destra, una a sinistra.

Circa due ore dopo il secondo atto."1"

Nos imaginamos un decorado poco real, de cartón, que servirá de fondo falso y pretencioso a una acción sólo pseudodramática donde los hombres interpretan la vida como personajes poco creíbles, marionetas, y en el que "Il Signore in grigio» sigue dirigiendo la obra, señalando de nuevo esa doble función: acción-espectador.

Esta salita tiene en escena una entrada abierta o cerrada por unas cortinas y una puerta sólo aparente simulada por otras cortinas iguales que intervienen en la obra como medio de incomunicación, impidiendo al personaje femenino la huida en un momento determinado, como imposibilidad de escapar al destino.

Las dos mesas, lo mismo que la mesa de la Sala del Telégrafo, marcarán la proximidad de los personajes presentes y la lejanía de los ausentes. En este acto, como a lo largo de toda la obra, existen además de los tres personajes que aparecen en escena otros personajes teatralmente existentes pero escénicamente ausentes que sólo conoceremos a través del diálogo, y que serán la causa del sufrimiento de los presentes. El tradicional triángulo amoroso marido-mujer-amante aparece distorsionado para representar cada uno de los tres personajes su propio drama, multiplicando así las combinaciones $^{17}$.

Entre los objetos que descubrimos en este tercer acto a través del diálogo y las acotaciones están la reluciente cristalería, las copas de champán que sirven a los tres personajes para brindar por los ausentes y a "Il Signore in grigio" para hallar la solución a su vida: la muerte.

Aparecerán como parte del decorado también unas flores que, en la última escena, materializan la presencia de los personajes ausentes, señalando una vez más ese dentro-fuera, cerca-lejos que delimita el espacio teatral: "garofani rossi», apasionados como el amor de la "Signora dalla volpe azzurra»; "viole del pensiero» melancólicas como "Il Signore a lutto" y "crisantemi» de muerte para "Il Signore in grigio». Serán éstos los signos

16 Rosso di SAN SECONDO, op. cit., p. 156.

17 Sobre este tema cfr. A. BARSotti, Pier $M^{\circ}{ }^{a}$ Rosso di San Secondo, Firenze, La Nuova Italia, 1978. 
explícitos de un proceso de transformación del amante lejano en objeto de culto.

En este tercer acto todo contribuye a la construcción de una realidad ficticia como instrumento para exorcizar la pasión, creando una atmósfera alucinante e hiperbólica, explendidamente realizada en la escena de los preparativos que llega a ser casi la celebración de un acto fúnebre.

La acción tiene lugar dos horas después del segundo acto y está estructuralmente condensada en movimientos de luz que van desde la penumbra de la Sala de Telégrafos a las lamparitas encendidas una hora después en la casa de «La Cantante» y de ahí a la lámpara central que ilumina la salita del restaurante.

La situación de la obra se desarrolla en el arco de tiempo de tres horas, con un ritmo adecuado a la concentración de un misterioso ritual: largas pausas, soñolientos silencios, etc...

El espacio va de un lugar público (Sala de Telégrafos) a otro privado (casa de "La Cantante»), y de ahí a otro más íntimo, aunque público (salita privada) protegido de la presencia de extraños que, por otra parte, es una constante en las obras de Rosso.

\section{EFECTOS SONOROS NO ARTICULADOS}

Dentro de los signos acústicos que se dan en escena como efectos sonoros no articulados por el actor están la música y el sonido.

La música es el único sistema de signos de los señalados por Kowzan que no aparece en Marionette, che passione!, sin embargo el sonido sí que está vivamente presente repetidas veces cumpliendo funciones bien determinadas.

En esta obra podemos distinguir dos formas de tratamiento del sonido. En primer lugar el silencio como "no sonido" cargado de significado ${ }^{18}$ : Rosso, explica él mismo, renuncia aquí al diálogo esencial, sabiendo que en el ambiente de su drama no hay sitio para discursos y razonamientos. Sólo son posibles el grito o el silencio que habla con los ojos, y en segundo lugar, la descripción de los sonidos como un recurso de intensa efectividad dramática. El sonido será el lenguaje que se intercale con la palabra, así, por

18 «L'uso sistematico del silenzio e del vuoto sembra caratteristica del teatro novecentesco, tendente a stabilire col pubblico un rapporto speciale (e diverso da quello tradizionale) che può andare dalla Verfremdung brechtiana all'aggresione o alla provocazione neurotica..." En F. FIDo, Guida al Goldoni, Torino, Einaudi, 1977, p. $116 n$. 
ejemplo, a lo largo de todo el primer acto el continuo sonido «della bollatura", de «i soldi per il pagamento» y el «ticchettio delle scarpette» de "La Cantante» en la Sala del Telégrafo contribuyen a crear ese ambiente soñoliento y monótono que ya hemos señalado, también a través de la monotonía del rítmico sonido del golpear constante del tampón y del taconeo de «La Cantante».

Otro sonido, la tos de «Il Signore in grigio» indicará su presencia antes de intervenir en el diálogo.

En el segundo acto "S'ode picchiare a una porta», ese "picchiare» está descrito en el diálogo como "grattare» evidenciando así los movimientos casi felinos ya que a un gato se asemeja en este acto «Il Signore in grigio».

Al final del tercer acto, en el momento culminante del drama, después del suicidio de "Il Signore in grigio», al final de la obra, a las palabras telegráficas de "Il Signore a lutto" «Risolto... Già risolto!» al silencio y soledad más atroz, acompañan risas escandalosas de mujeres que pasan por la calle, canturreos de alguien que pasa detrás de las cortinas y sonidos de cubiertos y platos que introducen de nuevo la realidad en escena. A pesar de todo, la vida continua su curso.

\section{APARIENCIAS EXTERIORES DEL ACTOR}

Otro aspecto dentro del análisis de las acotaciones, es el funcionamiento de los signos referidos al vestuario, los significantes producidos por la actividad ejercida sobre el actor: maquillaje, peinado, traje...

Así pues, podemos ver cómo el tiempo atmosférico «uggioso e piovigginoso» en el que Rosso sitúa la obra se introduce a través de la vestimenta de los personajes que van entrando: «Il Fattorino di prefettura», con «l'impermeabile stillante di pioggia». Más tarde entrará la «Signora dalla volpe azzurra» con «l'ombrello grondante di pioggia»e «Il Signore a lutto» e «Il Signore in grigio» con abrigo, sombrero y paraguas; todos ellos signos escénicos muy precisos que señalan el tiempo atmosférico y la estación del año.

Los personajes de Rosso no tienen nombre propio, es ésta una características del teatro expresionista que lo que busca son tipos más que individuos, por lo que tiende a evitar el nombre propio y a denominarlos por nombres comunes, son personajes individualizados a través de su vestimenta; el hombre sólo se reconoce por la ropa que lleva, es un fantoche deshumanizado por exceso de pasión o sufrimiento.

Rosso, a través del color, quiere reflejar la íntima conciencia de los protagonistas, la expresión de un estado de ánimo particular de angustia, de 
delirio; así «Il Signore in grigio» visualiza «il grigiore» de la propia existencia y de la propia razón, "Il Signore a lutto» nos muestra el luto de su propio ser y la "Signora dalla volpe azzurra" con su «abito di passeggio, quasi di viaggio", denota el deseo de viajar al sentirse abandonada, el deseo de huir, de cambiar, de provocar otros encuentros, otras posibilidades de vida.

Al «Signore in grigio" nos lo describe con facciones robustas, musculosas, "viso aperto, capigliatura folta gittata in dietro..."; mientras que «Il Signore a lutto» es sólo un nombre, sin descripción física, sin otras imágenes externas.

La «Signora dalla volpe azzurra» es «snella, elegante, senza ricercatezza, ha una volpe azzurra sulle spalle intorno al collo, guantata...». Y de todo su atuendo va a ser el guante olvidado de la Señora el objeto que sirva de pretexto para la continuación en el desarrollo de la acción.

Además de los tres protagonistas hay sólo otro personaje al que se alude, "Colui che non doveva giungere», del que nos dirá que es alto y robusto. «Pastrano abbottonato, capello in testa, ombrello».

Del resto no hay descripción física ni en las acotaciones ni en el diálogo, sin embargo sólo a través de la vestimenta que llevan deberán de dar al espectador la imagen de lo que representan, como es el caso de los dos "Operari» en el primer acto, los dos «Cameriere», "Una Mondana» y "Un Damerino" que intervienen en el tercer acto. En el proceso de condensación que (como otros géneros) ofrece el teatro, todo es funcional, todo tiene un valor de signo.

\section{EXPRESIÓN CORPORAL}

Pasaremos a analizar los signos «kinésicos» que pertenecen a la actividad del actor: mímica, gesto y movimiento, que acompañan siempre a los signos del texto dialogado.

En una obra en la que predominan las pausas y los silencios, no cabe duda que el conjunto más importante de significantes llegan al espectador a través de la expresión física.

Por los movimientos y gestos descritos por el autor en las acotaciones podemos ver cuál es la situación de angustia, indecisión y sufrimiento de los tres personajes, antes de que se evidencie en el diálogo: «Il Signore in grigio» fuma nerviósamente, cruza las piernas, «depone la penna che teneva in mano, accende una sigaretta, fuma a grandi boccate, butta la sigaretta, si prova di nuovo a scrivere, depone ancora la penna, si rovescia da un lato, 
poi dall'altro, fissa il pavimento, la punta delle scarpe; si tocca la fronte, il naso, il mento, si stringe la mani..." ${ }^{19}$.

«ll Signore a lutto» "stende il telegramma risolutamente; ma quando l'ha scritto, lo rilegge, e invece d'alzarsi per passarlo, lo ridepone lentamente sulla tavola, aggrotta le ciglia, rimane a pensare, immobile... lacera il suo telegramma, lo getta nel cestino, si alza, riprende l'ombrello e par che voglia uscire, però ritorna alla tavola.... ${ }^{20}$.

La «Signora dalla volpe azzurra» "prende una penna, si dispone a scrivere ma non scrive... Si mette a scrivere, ma si sofferma e tralascia ${ }^{21}$.

Vemos a estos tres personajes unidos por una situación dramática, aunque todavía no sepamos cuál es, de la que poco a poco "Il Signore in grigio» va a intentar salir para convertirse en director de las acciones de los otros y de las suyas.

Podemos ver sólo por los gestos y movimientos enunciados en las acotaciones cuál va a ser la actitud de los tres personajes que a su vez indicarán el punto en el que se encuentra su Pasión, que será el motor de cada uno de ellos: así, la «Signora dalla volpe azzurra» aparece «Fissa con occhi ebeti il vuoto / voce bassa / idiota, stupida / sussulto angoscioso / reprime singhiozzi nel fazzoletto / Ha un tremito, non risponde / Rimane a sedere ebete / gemendo straziata / tremando / d'un pallore mortale». Esta palidez mortal es un significante enormemente preciso, aunque dificil de conseguir por parte del actor, que veremos repetida al final de la obra "si sbianca» y en el rostro de «La Cantante»: «pallida, rivedendo invece l'altro nella memoria».

A través de estas descripciones observamos en la «Signora dalla volpe azzurra" una actitud de hipnosis que indica la primera fase del tormento provocado por un amor traicionado, la fijeza embobada que sigue inmediatamente a una ruptura, el vacio emotivo hacia el llanto. Esta sería la primera fase de la Pasión.

Rosso nos presenta al «Signore a lutto»: «voce tremante / fissità di pazzo, angosciosamente, disperatamente / scattando con un tremito di singhiozzi nella voce, / convulso / pianto disperato / tra i singhiozzi / nuovo assalto d'angoscia / La si stringe addosso come un naufrago alla tavolar. Sus gestos característicos son convulsivos, una breve expansión y una rápida contracción. El autor nos indicará en este personaje el segundo escalón de la pasión, esa ascensión exasperada que sucede a la apatía y que se manifiesta en los esfuerzos por rehacer el fracaso de su experiencia.

En la acotación que acompaña a la primera intervención dialogada de este personaje se incluye, como en muchas otras, una valoración del autor:

\footnotetext{
19 Rosso di SAN SECondo, op. cit., p. 133.

${ }^{20}$ Ibidem., p. 134.

21 Ibidem., p. 135.
} 
«sembra un uomo perfectamente ragionevole, anzi terribilmente lógico: soltando gli occhi hanno uno scintillio vitreo, in cui s'avverte l'anormalità del suo stato ${ }^{22}$, en esta acotación está encerrado todo su comportamiento a lo largo de la obra. Por una parte se presentará como Razonador desencantado y por otra prisionero y encadenado por el amor, figura en permanente contradicción que resume en si los atributos de sus interlocutores. Es el personaje con el que se identifica el autor, mitad cabeza y mitad cuerpo. En él podemos ver también reflejada la dicotomía entre Norte (razón) y Sur (pasión), señalada por Tilgher ${ }^{23}$, que será una constante en las obras de Rosso.

En los modos de actuación de «Il Signore in grigio» se expresa la última fase del progreso del mal, el amor sin salvación posible en el hombre. Una vez superadas las fases de abatimiento y absurda esperanza, del colapso y la convulsión (síntomas todavía vitales de una pasión), parece llegar a una condición de lucidez artificiosa, semejante a la mejoría antes de la muerte.

No todos los movimientos señalados en las acotaciones están ligados al diálogo; por ejemplo, «La Cantante» «Va per uscire a sinistra, sulla soglia si volge, non vista dall'amica fa un cenno ammonitore con la mano al Signore in grigio il quale la rassicura con un movimiento del capo" ${ }^{24}$.

También hay sonidos y palabras que se pronuncian en la obra y que sin embargo no están en el texto dialogado: «Ella mormora casi impercettibilmente: Buona sera» y más adelante «s'ode la Cantante che continua ad esortare: «Su, coraggio. Non è nulla».

En este segundo acto y también a través del gesto y el movimiento vemos el paso del amor-desolación al amor-juego en el que "Il Signore in grigio" se transforma en seductor evidenciando de nuevo el doble personaje al mismo tiempo eufórico y angustiado que encierra su personalidad. Termina por contagiar con sus gestos divertidos la escena, conduciendo un cortejo carnavalesco en el que erige en brazos a «La Cantante» a modo de trofeo: Vemos al «Signore in grigio» "Accenando passi di danza»...«le abbraccia le ginocchia e si alza portandola in braccio....apre le braccia, perché la Cantante scivoli a terra, ma le richiude subito ed ella è costretta a dargli un bacion" ${ }^{25}$.

Si el valor de las acotaciones es esencial en todo el texto, en el tercer acto son imprescindibles para que el espectador pueda ver el cuidado con el que «Il Signore in grigio» organiza la escena final del restaurante: la situación de las mesas $y$, sobre todo, la meticulosa preparación de éstas, la disposición de las sillas, de los cubiertos, de los personajes ausentes y presentes, de las flores, de las copas, etc...Vemos como «Il Cameriere» "Leva

22 Rosso di SAN SECONDo, op. cit., p. 141.

${ }^{23}$ A. Tilgher, «Rosso di San Secondo uomo del Sud», en AA.VV., Letteratura italiana. Novecento, III, Milano, Marzoratti, 1980, pp. 2548-2550.

${ }^{24}$ Rosso di SAN SECONDO, op. cit., p. 150.

2s Ibidem., p. 154. 
il quarto coperto dalla tavola destra e ne toglie la sedia che accosta alla parete: piega le altre tre sedie contro i coperti che rimangono" e «Il Signore in grigio" «Alla Signora dalla volpe azzurra...Fa posto alla tavola di sinistra. La Signora dalla volpe azzurra siede in modo da rivolgere il volto al publico...Indica il posto alla destra della signora. Il Signore a lutto si siede. Il Signore in grigio, sedendo a sinistra della dama ${ }^{26}$.

Más adelante entra "Il Cameriere» con un ramo de flores que «Il Signore in grigio" de pie, con las flores en la mano, va repartiendo en la mesa de los ausentes, en la que al final de la obra sólo permanecerán las «viole del pensiero», ya que la "Signora dalla volpe azzurra» una vez descubierta por su amante «passando accanto alla tavola dei fiori, pur barcollando, ha modo di stendere una mano, prendere i garofani rossi e, uscendo, ficcarli tra il petto e il pastrano di «Colui che non doveva giungere» ${ }^{27}$. Mientras que «Il Signore in grigio" "prende i crisantemi. Li sparge per terra come sopra una bara" anunciando asi su propio epitafio ya que, inmediatamente, "Mentre parla lentamente trae di tasca il portafoglio, ne cava una cartina, l'apre, s'avvicina alla tovola e ne versa il contenuto in un mezzo bicchiere di champagne, battendo con il dito il pezzetto di carta, perché la polverina caschi interamente $\rangle^{28}$.

Ha sido invención de "Il Signore in grigio" esta espectacular puesta en escena (de nuevo el teatro dentro del teatro) que constituye así la última parte del drama con una fantasía morbosa, que él mismo cierra con su propia muerte.

En este acto aparecerá la muerte bajo diversas formas: «Il Signore in grigio» muere, la "Signora dalla volpe azzurra» vuelve con el amante para someterse de nuevo al papel de mujer-objeto y «Il Signore a lutto» perderá toda esperanza de evasión.

Mediante el movimiento, en el juego fuera / dentro de los personajes ${ }^{29}$ podemos ver cómo en la obra de Rosso el mundo externo puede penetrar de repente y turbar los proyectos visionarios de los protagonistas obligándoles a repentinos retornos a la realidad objetiva y en el espacio visible. Las marionetas se ven interrumpidas continuamente por grupos que irrumpen de fuera («Fattorini, operai, signore, signora e fanciulla...» en el primer acto) que envían telegramas con noticias sobre muerte, nacimiento, matrimonio, acontecimientos éstos básicos de la vida humana. En el segundo acto irrumpen las bailarinas y en el tercero los camareros, «la Mondana e il Damerino» y finalmente "Colui che non doveva giungere».

${ }^{26}$ RossSo di SAN SECONDO, op. cit., p. 157.

${ }^{27}$ Ibidem, p. 163.

${ }^{28}$ Ibidem., p. 164.

29 Tema éste bien estudiado por C. PÉrez Gállego, «Dentro-fuera y presente-ausente en teatro", en Semiología del teatro, Barcelona, Planeta, 1975. 
La entrada de todos estos personajes, desde el primer acto, aisla todavía más a los tres protagonistas de su contexto social.

Hemos visto cómo las acotaciones en esta obra, dejando a un lado su función referencial, adquieren un relieve cada vez más eminente respecto al diálogo, hasta llegar a configurarse como necesaria integración y asumir, como dice Chiarini una legítima dignidad de «lectura».

Las indicaciones escénicas pierden su función de trazo instrumental para hacerse estructura portadora de su poética, asumiendo un espesor semántico interno de decisiva importancia. 